Session 2164

\title{
Recruiting a New Generation of Ceramic Engineers
}

\author{
William G. Fahrenholtz, Richard K. Brow, and Amanda L. Young \\ Ceramic Engineering Department \\ University of Missouri-Rolla
}

\begin{abstract}
Students at the University of Missouri-Rolla (UMR) were awarded a grant to develop kits that could be used by high school science teachers to promote interest in ceramic materials to their students. The proposal was funded by the Associated Glass and Pottery Manufacturers Association (AGPMA) with the intent of increasing the visibility of Ceramic Engineering as a career to high school students.

As part of the project, UMR undergraduates are developing and testing self-contained kits for distribution to high schools. The kits will contain all of the materials and supplies needed for high school teachers to perform experiments and controlled demonstrations that illustrate important scientific principles in an entertaining manner. Laboratory exercises based on slip casting and glass melting have been prepared for the kits. These hands-on activities give a basic understanding for what ceramic materials are and how they are produced. They emphasize the application of science (chemistry and physics) to form common raw materials into useful products. At the end of the funding cycle, an example kit, written instructions, and an interactive digital video disk (DVD) will be delivered to AGPMA for distribution to high schools.

The kits will include materials for slip casting clay-based ceramics, glass making, a ceramic superconductor demonstration, and a space shuttle tile demonstration. The slip casting materials include pre-made slips that will fire to various colors. The kit also contains molds and glazes. The glass making kit includes instructions, raw materials, and supplies needed to make watercorn syrup-sugar analogues to the modifier-intermediate-glass former roles of the components in soda-lime-silicate glasses. Each kit also comes with an $\mathrm{YBa}_{2} \mathrm{Cu}_{3} \mathrm{O}_{7-\delta}$ pellet and a magnet for demonstration of the Meissner effect. Finally, the kits contain a space shuttle tile and instructions for demonstrations of the insulating power of this fascinating material.
\end{abstract}

Proceedings of the 2003 American Society for Engineering Education Annual Conference \& Exposition

Copyright (C) 2003, American Society for Engineering Education 


\section{Introduction}

Materials-related engineering departments at most universities recognize that recruiting is necessary to attract undergraduates into the field. These recruiting activities increase name recognition and visibility of this relatively small engineering discipline among high school students. A variety of outreach materials for recruiting activities are available to universities through technical societies such as the American Ceramic Society. ${ }^{1}$ These materials tend to focus on demonstrations such as levitation of a magnet by a ceramic (high temperature) superconductor or the thermal insulating properties of space shuttle tiles. While the demonstrations are captivating and do promote interest in the field, they do not provide teachers at the elementary or secondary education levels with content for their classes. The goal of this program is to provide a combination of interesting demonstrations and in-class experiments that can be carried out by high school teachers in the facilities available in most public schools. The demonstrations are meant to foster classroom discussions of ceramic materials that promote inquiry-based learning. The experiments will provide the students with hands-on learning opportunities while the kits will provide the resources and background materials necessary to setup and understand the experiments. Further, the finished AGPMA kits will contain an interactive DVD that will provide explicit instructions for using the kits along with explanations of the science behind the experiments. The DVD will also contain information on educational opportunities and descriptions of careers in Ceramic Engineering. Prior to delivery to AGPMA, the kits will be tested by UMR. Testing of the kits will proceed in two steps. The initial phase of testing has included visits to local and regional classrooms. On these visits, UMR students perform controlled demonstrations and then use feedback from the students and teachers to assess the level of detail that will be required in the instructional materials so that the teachers and students can understand what they are doing. In the second phase of testing, UMR will send prototype kits to high schools. Feedback will be collected from teachers in this phase and will be used to finalize the instructional materials that accompany the kits. The purpose of this paper is to describe the AGPMA recruiting kit.

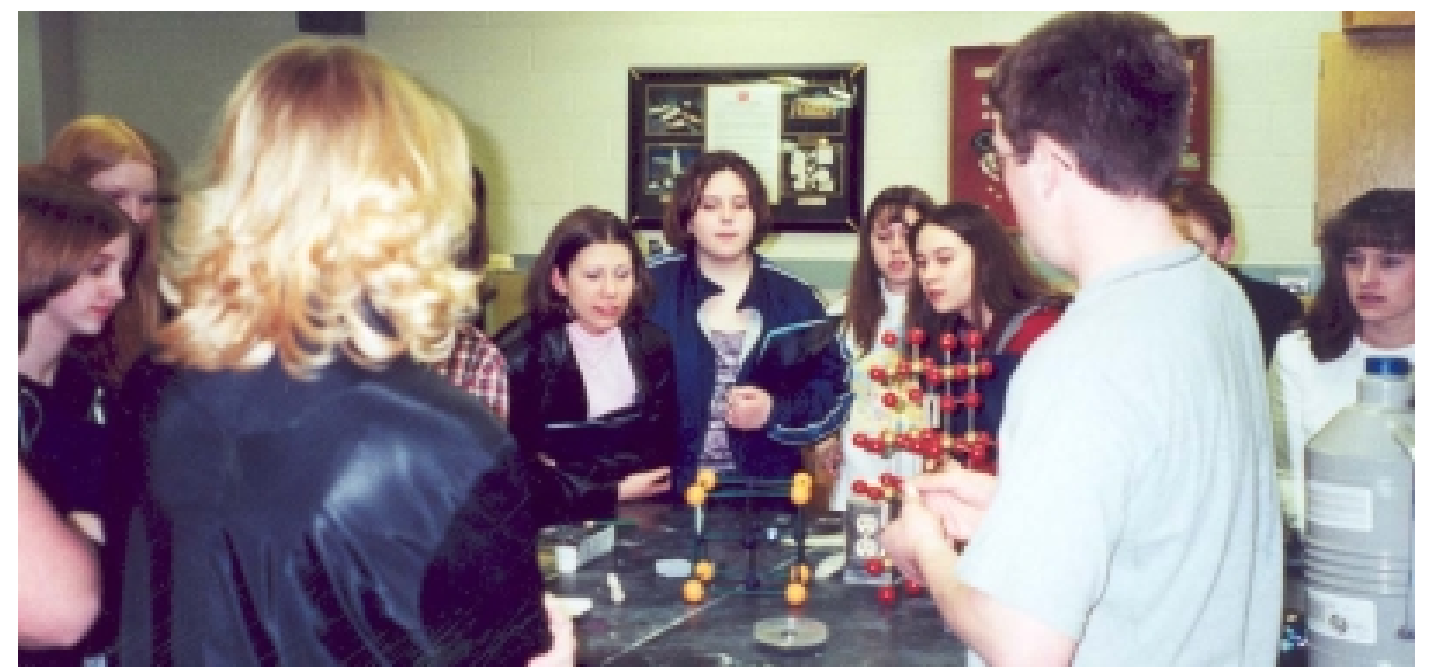

Figure 1. A typical laboratory tour for a high school class visiting the Ceramic Engineering department at the University of Missouri-Rolla. 


\section{Forming Clay-Based Ceramics by Slip Casting}

When asked to identify ceramic materials, most high school students (and the general public) immediately name traditional ceramics such as flowerpots, plates, sinks, and refractories. These clay-based objects are familiar to everyone because they are stand-alone consumer products that are produced in high volume. In contrast, most educational and employment opportunities in ceramic engineering involve technical ceramics which are more difficult to identify because technical ceramics serve as functional components that are hidden inside consumer products such as televisions and computers. Many of the principles behind the forming methods used to produce technical ceramics can be demonstrated with traditional materials and forming methods. Slip casting will be used in the AGPMA kits. A slip is a stable suspension of clay particles (feels slippery between the fingers). For slip casting, a slip is poured into a porous mold and a solid layer forms due to the build up of particles on the mold wall as water from the suspension is drawn into the mold. Thin walled, hollow objects such as decorative figurines are produced this way as are complex parts such as toilets and sinks.

Clays are used as raw materials for most traditional ceramics due to their abundance, low cost, and ease of forming. With their long history and their ubiquity, traditional ceramics and the methods used to form them can be used to demonstrate concepts from thermodynamics and kinetics. As discussed in more detail below, the activities suggested in the AGPMA kit are described in ways that can be understood by high school teachers who will then explain them to high school students. The AGPMA kits will contain all of the supplies needed for high school classes to form traditional ceramics by slip casting, including plaster molds and pre-mixed clay suspensions. After casting, the ceramics using pottery kilns that might be available in the school art departments or by accessing facilities at local art centers or ceramics dealers. The instructions provided with the kits will explain how to use the pre-mixed slips, how to formulate other slips from commonly available raw materials, and how to cast, dry, fire, and glaze objects. In addition, the AGPMA kits will also contain information on scientific experiments that can be performed. In the case of slip casting, experiments related to diffusion and plastic deformation can be performed and will be described in detail in the DVD. A brief description of the diffusion related experiments follows.

Diffusion is the movement of solute atoms through a solvent due to a gradient in chemical potential. Materials engineers and scientists apply diffusion theory to understand and control the kinetics of reactions at high temperatures and changes in composition across interfaces. Some common examples of diffusion are the migration of odors across a room through stagnant air, the movement of water through underground rocks, and the motion of a dissolving colorant through a stagnant solution. Although the mathematics of diffusion are exceedingly complex, diffusion can easily be explained in a qualitative manner with illustrations familiar to students. The traditional ceramic forming method of slip casting can be used to perform some simple diffusion experiments that illustrate the principles of kinetics. One experiment that is suggested is to measure the thickness of pieces cast for times ranging from a few minutes to nearly an hour. The data can then be analyzed by plotting thickness as a function of time and comparing that to a plot of thickness as a function of the square root of time. The square root of time plot should result in 
a straight line as would be expected for any diffusion controlled process. Two examples where diffusion plays a role in everyday life include determining the thickness of packaging material required to keep foods fresh (e.g., the thickness of plastic wrap needed to protect meat from oxidation during its shelf life) and the cooking time for dried foods (e.g., how much longer does it take to cook pasta that is twice as thick). To build on the observations, students can be asked to research and prepare short reports on other diffusion-controlled phenomena such as the design of gas separation membranes, the time and temperature of heat treatment for semiconductor processing, the kinetics of thermal decomposition of salts, or the operation of the oxygen ion conductors in solid oxide fuel cells according to their interests. The students can be instructed to differentiate between processes controlled by diffusion and those whose rates are controlled by other physical phenomena. For example, are easily observed phenomena such as the sedimentation of particles from a suspension or the time required for gelatin to set controlled by diffusion? To explore the finer points of diffusion, students can be asked to speculate on the effect of particle size on rate of slip casting and the effect of particle size on the packing density of a slip cast layer.

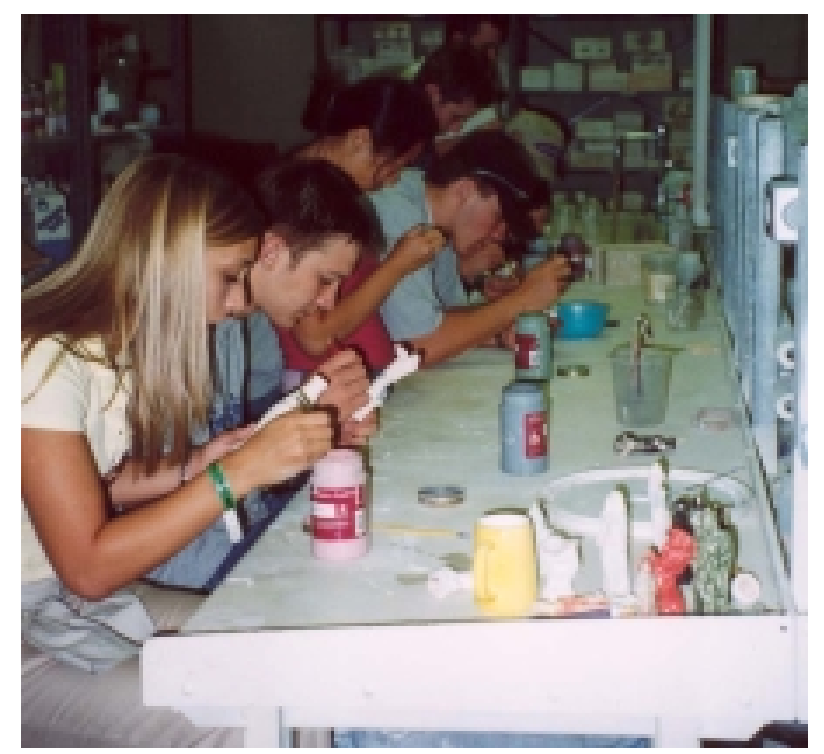

Figure 2. Tour groups participating in slip casting activities at UMR.

\section{Fabrication and Properties of Glass}

Glasses are aesthetically pleasing and technologically important non-crystalline solids. Common silicate glasses are optically transparent. Because of the unique thermal, rheological, and optical properties of glasses, glass formation provides many interesting opportunities for experimentation. Unfortunately, most inorganic glasses require temperatures in excess of $1000^{\circ} \mathrm{C}$ for fabrication. In addition to the danger inherent to high temperature operations, most high schools do not have the facilities, refractory crucibles, and protective equipment required to melt and pour silicate glasses (Figure 3). However, low melting, organic analogues exist for silicate glasses. Organic glasses can be prepared using mixtures of table sugar, corn syrup, and water, which act as the glass former, intermediate, and network modifier, respectively. ${ }^{2}$ The processing temperature for these sugar-based organic glasses is around the boiling point of water, 
$100^{\circ} \mathrm{C}$. Organic glasses can be prepared in standard laboratory beakers or household cookware using hot plates that are available in the chemistry laboratories of most high schools. The glass activities in the AGPMA kit are based on a series of experiments developed by a father-daughter team. In addition to their low temperature forming, these glasses are non-toxic (edible and quite delicious, in fact), which eliminates concerns of ventilation during processing and waste disposal. The decision to use organic analogues to silicate glasses in the AGPMA kits was made after exploring other options including the use of highly fluxed borate and silicate compositions. Even with high modifier concentrations, the processing temperatures of these glasses were still around $1000^{\circ} \mathrm{C}$. Most high schools do not have appropriate furnaces to prepare glasses at these temperatures, so the kits would have to include a high temperature furnace. Even inexpensive furnaces capable of melting only a few tens of cubic centimeters of glass were beyond the budget of this project. In addition, sending potentially dangerous equipment to high schools could create concerns about safety and liability that would limit distribution of the kits.

As suggested by Jain and Jain, a variety of glass-related activities can be performed in the sugarcorn syrup-water system. ${ }^{2}$ The AGPMA kit will include the ingredients to make the glasses along with a pan for melting and beakers for pouring glasses. In addition, the kits will contain information on the structure of crystalline compounds and how they differ from glasses. The suggested experiments will follow closely from those suggested by Jain and Jain. The experiments will include growth of sugar crystals from the melt and preparation of samples to examine the effects of additions of intermediate and modifiers on the solidification temperature and properties of the final glass. Beyond the fun of preparing and examining the different compositions, instructional materials will suggest other activities such as developing phase diagrams (e.g., what is the limit of water solubility in sugar as a function of temperature) and drawing fibers. Some other exercises for the students will include researching the history of glass processing, comparing and contrasting the sugar glasses to silicate glasses, and writing reports on high-tech applications for glasses.

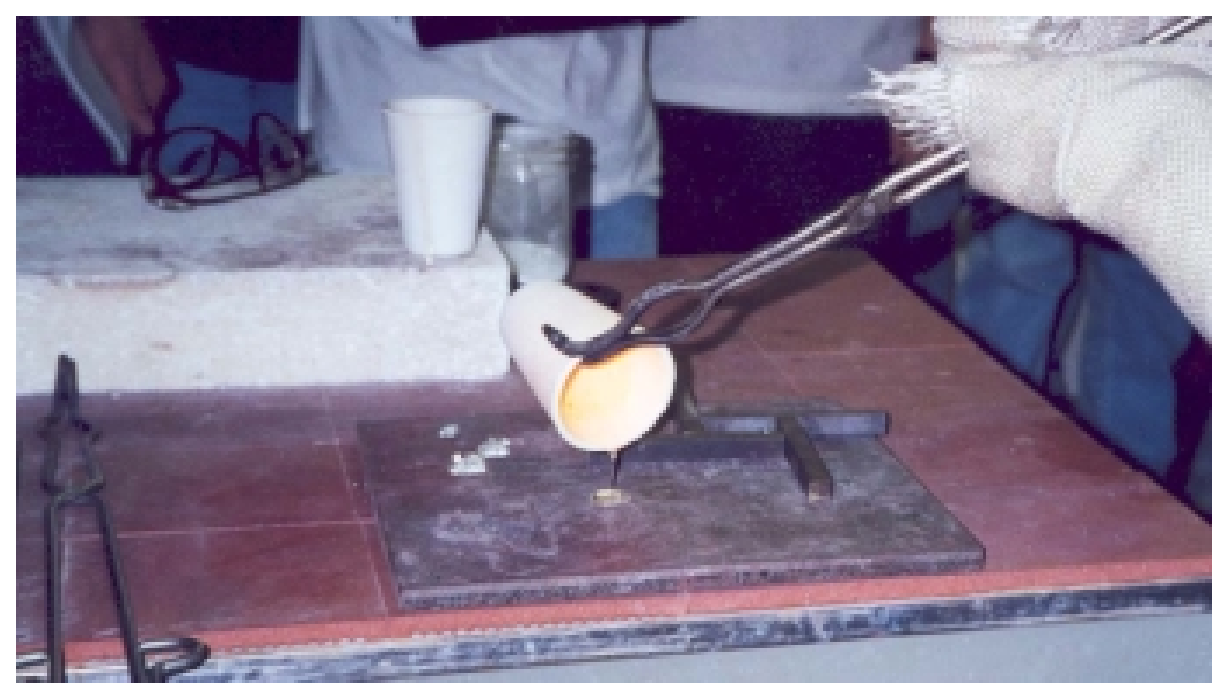

Figure 3. Pouring of a high temperature borate-based glass. 


\section{Superconductor Demonstration}

High temperature superconductivity has captivated the imagination of research scientists and the general public for more than fifteen years. Images of levitating magnets, continuous advancements in critical temperature, and speculation on applications ranging from loss-free power transmission to high-speed mag-lev trains have kept superconductivity in the news. The AGPMA kits will include the supplies needed to perform a superconducitivty demonstration along with a thorough explanation of the physical phenomenon that causes the magnet to levitate, the Meissner effect. The kit will include an $\mathrm{YBa}_{2} \mathrm{Cu}_{3} \mathrm{O}_{7-\delta}$ pellet, a rare earth magnet, a Petri dish, non-conductive tweezers, and a small dewer for holding liquid nitrogen. The instructions will provide suggestions for suppliers of liquid nitrogen, give guidance for the demonstration, and suggest other activities to use the liquid nitrogen. Liquid nitrogen is commonly available from welding suppliers, university chemistry departments, and some medical practices. Many of these may be willing to donate small quantities to the teachers for use at school. A warning of the dangers of liquid nitrogen will be included in the instructions.

The superconductor demonstration is simple. First, the superconducting pellet is cooled below its critical temperature, around $90 \mathrm{~K}$ for $\mathrm{YBa}_{2} \mathrm{Cu}_{3} \mathrm{O}_{7-\delta}$, by immersion in liquid nitrogen. Then, the magnet can be set above the pellet and left stationary or spun. The Meissner effect is explained in terms of the nature of electo-magnetism; a perfect conductor of electricity is a perfect rejecter of magnetic field. This is in contrast to the type of repulsion exhibited by like poles on different magnets, giving superconducting systems several benefits for applications such as levitating trains. Details for other experiments that can be performed with liquid nitrogen will also be included with the instructions including immersion of balloons, shattering frozen objects, and eating frozen marshmallows. Immersion of a small balloon in liquid nitrogen can be used to demonstrate the ideal gas law. Students can calculated the volume/pressure of the gas in the balloon at room temperature and the liquid nitrogen temperature $(77 \mathrm{~K})$. When immersed, the balloon will collapse to a small fraction of its original volume, which provides a great visual accompaniment to the introduction of Charles' law in high school chemistry. Some common objects that can be shattered after immersion in liquid nitrogen include racquet balls, flowers, and pennies made after 1982 (the year the U.S. Mint began making them from zinc instead of copper). The accompanying explanation can be simple (cold things become brittle or ceramic like) or more complex (glass transition temperature in case of polymers and brittle-toductile transitions in metals), depending on the experience of the instructor. Finally, the tours at UMR always conclude consumption of frozen marshmallows. Like other polymers, marshmallows (sugar-based glassy polymer) become brittle below their glass transition temperature. When eaten, the frozen marshmallows shatter in the mouth and cause the eater's breath to freeze, similar to what happens when you exhale outside on a cold day. The explanation that follows can focus on the marshmallow or the breath.

\section{Space Shuttle Tile Demonstration}

The fleet of space shuttle orbiters used by NASA is one of the most visible examples high performance ceramic materials. The ceramic tiles used for insulation on the orbiters are 
lightweight and durable. Development of silica-based thermal protection tiles enabled a revolutionary step forward in spacecraft design from the single-use capsules of the Mercury, Gemini, and Apollo programs to the reusable orbiters. The older vehicles used ablative thermal protection materials that were consumed during reentry. Though some maintenance is required, the tiles on the shuttle are designed to withstand dozens of flights.

The insulating power of these tiles can be demonstrated in the classroom with a propane torch. Although a high temperature flame is used, the demonstration can be conducted safely in a controlled setting. The usual set-up has a student heat one side of a tile while hold his/her hand against the other (Figure 4). The torch is extinguished after the front side has begun to glow red. The tile is held with the cold face up to allow other the students to feel that the unheated surface has remained at room temperature. The accompanying explanation includes a description of the three common mechanisms of heat transfer (conduction, convection, and radiation). Sample questions such as "how is heat transferred from the torch to the tile surface?" and "how is heat transferred through the tile?" will be provided to the teachers along with answers. In addition, other everyday situations where heat transfer is important (cooking on the stove at home, heating systems for buildings, the sun) are discussed along with potential research assignments for the class.

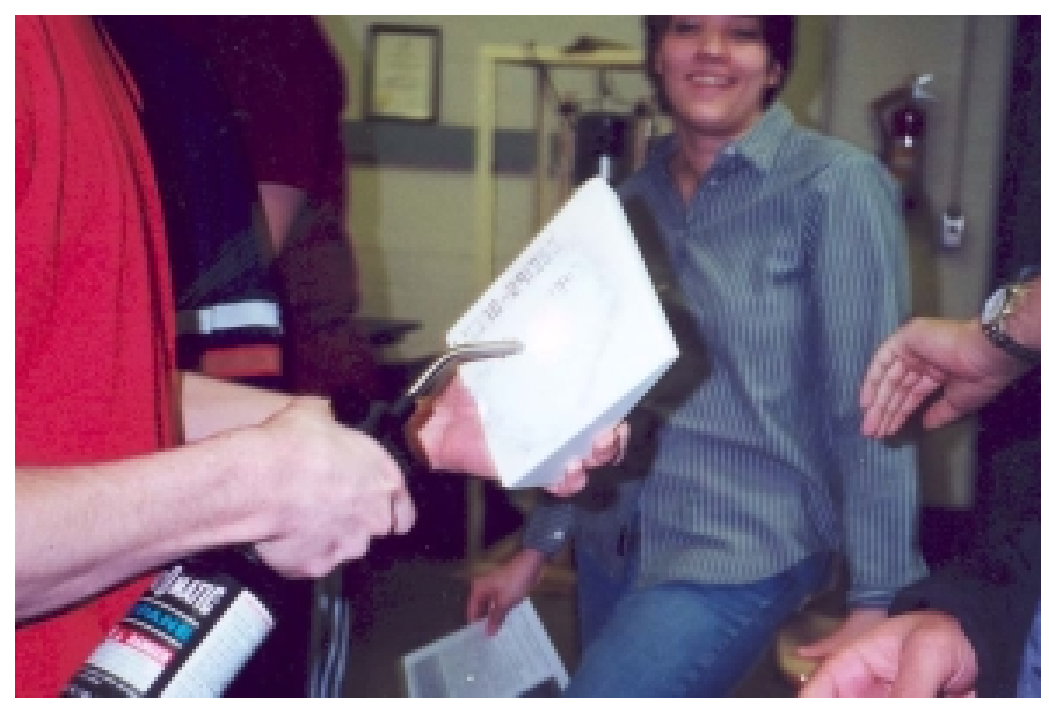

Figure 4. Demonstration of the insulating power of space shuttle tiles.

\section{Interactive DVD}

A DVD will accompany the AGPMA kits. The DVD will include detailed instructions for the experiments and demonstrations. In addition, the DVD will list of additional resources available for high school teachers (a partial list is included in Table 1), describe additional activities that can be performed, provide follow-on assignments, give information on careers for ceramic engineers, and provide contact information for UMR faculty willing to visit classrooms or answer questions by email. 
Session 2164

Table 1. Partial list of additional materials related resources for high school teachers.

\begin{tabular}{|l|l|l|}
\hline \multicolumn{1}{|c|}{ Name } & \multicolumn{1}{c|}{ Description } & \multicolumn{1}{c|}{ Web Location } \\
\hline Science on wheels & Simple materials demonstrations & www.acers.org/outreach/precollege.asp \\
\hline MAST modules & Materials-related lab exercises & matse1.mse.uiuc.edu/ tw/ \\
\hline ACerS sample kit & Samples of ceramic materials & www.acers.org/outreach/precollege.asp \\
\hline Alfred Univ. & How ceramics function & jobs.alfred.edu/ misture/nsf_k12.html \\
\hline UMR & Faculty and student contact info & www.umr.edu/ ceramics \\
\hline
\end{tabular}

\section{Plans for Distribution}

The AGPMA kits are being developed with the assistance of high schools in the Rolla area. During kit development, UMR students will make class room visits to test the materials in front of high school students and teachers. Next, prototype kits will be assembled and distributed to high schools for further refinement. Once the final experiments and demonstrations are assembled and the DVD is prepared, the kit will be sent to AGPMA for reproduction and distribution. In addition, UMR will continue to distribute the kits to high schools in Missouri and make visits to the schools to recruit future generations of ceramic engineers.

\section{Bibliography}

1. http://www.acers.org/outreach/awareness.asp

2. H. Jain, and I.H. Jain, "Discovering the Science and Technology of Glass Formation from Candy Making," Proceedings of the 2002 ASEE Annual Conference and Exposition, June 18-23, 2002, Montreal, Canada.

WILLIAM G. FAHRENHOLTZ

William G. Fahrenholtz is an assistant professor of Ceramic Engineering at UMR. He teaches two sophomore level laboratories and required lecture courses in x-ray diffraction and thermodynamics. Dr. Fahrenholtz is active in ceramics research and has published over 30 technical papers. He also coordinates a math and science competition for high school students in Missouri, the Worldwide Youth in Science and Engineering Academic Challenge.

\section{RICHARD K. BROW}

Richard K. Brow is professor and chair of the Ceramic Engineering Department at UMR. He teaches a sophomore level class on glass properties and structure, a senior level class in glass technology, and graduate classes on optical properties and glass science. Dr. Brow is an internationally recognized researcher and has won numerous awards in glass science. He is a past chair of the Glass and Optical Materials division of the American Ceramic Society.

AMANDA L. YOUNG

Amanda L. Young is a December 2002 graduate of the Ceramic Engineering department at UMR. She is currently pursuing her M.S. degree in Ceramic Engineering at UMR studying the fabrication of multi-layer ceramic capacitors using co-extrusion processing.

Proceedings of the 2003 American Society for Engineering Education Annual Conference \& Exposition Copyright (C) 2003, American Society for Engineering Education 